\title{
A strong upper limit on the pulsed radio luminosity of the compact object 1RXS J141256.0+792204 (Research Note)
}

\author{
J. W. T. Hessels ${ }^{1}$, B. W. Stappers ${ }^{1,2}$, R. E. Rutledge ${ }^{3}$, D. B. Fox ${ }^{4}$, and A. H. Shevchuk ${ }^{4}$ \\ 1 Astronomical Institute "Anton Pannekoek", University of Amsterdam, Kruislaan 403, 1098 SJ Amsterdam, The Netherlands \\ e-mail: jhessels@science.uva.nl \\ 2 Stichting ASTRON, Postbus 2, 7990 AA Dwingeloo, The Netherlands \\ e-mail: stappers@astron.nl \\ 3 Department of Physics, McGill University, 3600 University, H3A 2T8 Montreal, QC, Canada \\ e-mail: rutledge@physics.mcgill.ca \\ 4 Department of Astronomy \& Astrophysics, 525 Davey Laboratory, Pennsylvania State University, University Park, PA 16802, USA \\ e-mail: dfox@astro.psu.edu, ahs148@psu.edu
}

Received 21 July 2007 / Accepted 11 October 2007

\section{ABSTRACT}

\begin{abstract}
Context. The ROSAT X-ray source 1RXS J141256.0+792204 has recently been identified as a likely compact object whose properties suggest it could be a very nearby radio millisecond pulsar at $d=80-260 \mathrm{pc}$.

Aims. We investigated this hypothesis by searching for radio pulsations using the Westerbork Synthesis Radio Telescope. Methods. We observed 1RXS J141256.0+792204 at 385 and $1380 \mathrm{MHz}$, recording at high time and frequency resolution in order to maintain sensitivity to millisecond pulsations. These data were searched both for dispersed single pulses and using Fourier techniques sensitive to constant and orbitally modulated periodicities.

Results. No radio pulsations were detected in these observations, resulting in pulsed radio luminosity limits of $L_{400}^{\max } \approx$ $0.3(d / 250 \mathrm{pc})^{2} \mathrm{mJy} \mathrm{kpc}{ }^{2}$ and $L_{1400}^{\max } \approx 0.03(d / 250 \mathrm{pc})^{2} \mathrm{mJy} \mathrm{kpc}^{2}$ at 400 and $1400 \mathrm{MHz}$ respectively.

Conclusions. The lack of detectable radio pulsations from 1RXS J141256.0+792204 brings into question its identification as a nearby radio pulsar, though, because the pulsar could be beamed away from us, this hypothesis cannot be strictly ruled out.
\end{abstract}

Key words. stars: neutron - stars: pulsars: general

\section{Introduction}

Recently, Rutledge et al. (2007), hereafter RFS07, have identified the X-ray source 1RXS J141256.0+792204 (from the ROSAT All-Sky Survey Bright Source Catalog) as having an X-ray to optical flux ratio $F_{\mathrm{X}}(0.1-2.4 \mathrm{keV}) / F_{\mathrm{V}}>$ $8700(3 \sigma)$. Such a high ratio is strong evidence that 1RXS J141256.0+792204, dubbed "Calvera" by RFS07, is a compact object. RFS07 consider several specific source classes to explain 1RXS J141256.0+792204's X-ray spectrum and luminosity: an X-ray dim isolated neutron star (INS), an anomalous X-ray pulsar (AXP), a compact central object (CCO), or a nearby radio pulsar. Based on careful comparison of 1RXS J141256.0+792204's properties with the canonical features displayed by these different classes of neutron star, RFS07 conclude that the most likely explanation is that 1 RXS J141256.0+792204 is a very nearby radio pulsar $(d=$ $80-260 \mathrm{pc}$ ) similar to the radio millisecond pulsars (MSPs) residing in the globular cluster 47 Tuc.

We have investigated this interpretation by conducting sensitive searches for radio pulsations using the Westerbork Synthesis Radio Telescope (WSRT) in the Netherlands. No pulsations were found by these searches, and we use these nondetections to place strong limits on the pulsed radio luminosity of 1RXS J141256.0+792204. These limits bring into question the interpretation of this object as a nearby radio pulsar.

\section{Observations and analysis}

We observed 1RXS J141256.0+792204 a total of 4 times with WSRT at central observing frequencies of 385 and $1380 \mathrm{MHz}$ and with integration times of 0.5 or $1 \mathrm{~h}$ (Table 1). All observations were centered on J2000 coordinates RA $=14: 12: 55.51$, $\operatorname{Dec}=+79: 22: 06.7\left(l=118.3^{\circ}, b=37.0^{\circ}\right)$. WSRT was used in its tied array mode with $1425-\mathrm{m}$ dishes phased together to form a $\sim 43 / 12^{\prime \prime}$ wide beam at $385 / 1380 \mathrm{MHz}$, with a gain of $1.2 \mathrm{~K} / \mathrm{Jy}$. The most precise X-ray position available, which is derived from Chandra/HRC-I observations of the source taken by RFS07, falls well within the WSRT beam. We recorded data using the PuMa I pulsar backend (Voûte et al. 2002), which provided $0.16 / 0.32-\mathrm{MHz}$ channels, $10 / 80 \mathrm{MHz}$ of bandwidth, and $102.4 / 102.4 \mu$ s sampling at $385 / 1380 \mathrm{MHz}$. We made multiple, multi-frequency observations because of potential scintillation. If 1RXS J141256.0+792204 is indeed very close, then it likely has a very low DM $\left(\$ 10 \mathrm{pc} \mathrm{cm}^{-3}\right)$, and could strongly scintillate. The $385-\mathrm{MHz}$ data also gave high sensitivity in the event that 1 RXS J141256.0+792204 is a very steep spectrum source. The resulting intra-channel dispersive smearing in these configurations, scaled to a dispersion measure (DM) of $25 \mathrm{pc} \mathrm{cm}^{-3}$, is $t_{\mathrm{DM}}=0.6\left(\mathrm{DM} / 25 \mathrm{pc} \mathrm{cm}^{-3}\right) \mathrm{ms}$ at $385 \mathrm{MHz}$ and merely $t_{\mathrm{DM}}=22\left(\mathrm{DM} / 25 \mathrm{pc} \mathrm{cm}^{-3}\right) \mu$ s for the $1380-\mathrm{MHz}$ observations. At the high Galactic latitude of the source, interstellar scattering is unlikely to add significant additional smearing. The resolution 
Table 1. Summary of observations.

\begin{tabular}{cccccc}
\hline \hline $\begin{array}{c}\text { Epoch } \\
(\mathrm{MJD})\end{array}$ & $\begin{array}{c}\text { Int. Time } \\
(\mathrm{s})\end{array}$ & $\begin{array}{c}\text { Freq. } \\
(\mathrm{MHz})\end{array}$ & $\begin{array}{c}\text { Band. }^{a} \\
(\mathrm{MHz})\end{array}$ & $\begin{array}{c}\text { Samp. } \\
\mu \mathrm{s}\end{array}$ & $\begin{array}{c}S_{\max }{ }^{b} \\
(\mathrm{mJy})\end{array}$ \\
\hline 54217.98 & 1800 & 385 & $10 / 0.16$ & 102.4 & 4 \\
54218.01 & 1800 & 1380 & $60 / 0.32$ & 102.4 & 0.4 \\
54218.89 & 1800 & 1380 & $60 / 0.32$ & 102.4 & 0.4 \\
54245.74 & 3600 & 1380 & $60 / 0.32$ & 102.4 & 0.3 \\
\hline
\end{tabular}

a Total usable bandwidth/channel bandwidth.

${ }^{b}$ For the observation at $385 \mathrm{MHz}$ this is the upper limit on $400-\mathrm{MHz}$ flux density $S_{400}$. For the other three observations at $1380 \mathrm{MHz}$, this is the upper limit on the $1400-\mathrm{MHz}$ flux density $S_{1400}$.

of the $1380-\mathrm{MHz}$ data was more than adequate to be sensitive to an MSP with spin period $P_{\text {spin }} \gtrsim 0.7 \mathrm{~ms}$ and DM up to the highest trial DM we searched $\left(100 \mathrm{pc} \mathrm{cm}^{-3}\right)$. In the $385-\mathrm{MHz}$ data, we were sensitive to millisecond periodicities as well, as long as the DM was less than $\sim 25 \mathrm{pc} \mathrm{cm}^{-3}$.

The DM and spin period of this source are unknown and thus we must search over these parameters. To search over DM space, we constructed trial timeseries dedispersed in the range $\mathrm{DM}=0-100 \mathrm{pc} \mathrm{cm} \mathrm{cm}^{-3}$, with a spacing of $0.2 / 1 \mathrm{pc} \mathrm{cm}^{-3}$ for the $385 / 1380 \mathrm{MHz}$ data. Integrating to the edge of the Galaxy, the maximum predicted DM in the direction of 1RXS J141256.0+792204 is $45 \mathrm{pc} \mathrm{cm}^{-3}$ (from the NE2001 free electron density model of the Galaxy, Cordes \& Lazio 2002). Thus, even if it is distant, it is very unlikely that 1RXS J141256.0+792204 has a DM outside our search range. Radio frequency interference (RFI) was removed from the data both by eliminating some of the spectral channels before dedispersion and by removing known interference peaks, or "birdies", in the Fourier power spectrum. Each of these timeseries was then searched for periodicities using a Fourier-based technique and also checked for strong single pulses. As the majority of MSPs are in binaries, we employed searches that are sensitive to orbitally modulated periodicities by encorporating a further search over frequency drift in the Fourier domain. Overlapping sections of the timeseries, each 15-min in length, were also searched to improve sensitivity to the shortest orbital periods. To perform these searches, we used the package PRESTO ${ }^{1}$ (see Ransom et al. 2002, for a description of some of the search techniques included in this package). Overall, our search method was very similar, both in terms of the techniques and specific software used, to that presented in greater detail in e.g. Hessels et al. (2007).

The resulting candidate lists were examined by eye and automatically sifted in order to identify periodicities that occur at several trial DMs, peak in signal strength at non-zero DM, and occur in multiple observations. We folded the raw data at the periodicity, acceleration, and DM of potentially interesting candidates in order to further investigate whether they constituted likely astronomical signals or just RFI. As the predicted DM for a pulsar in this direction at $d<260 \mathrm{pc}$ is only $<3 \mathrm{pc} \mathrm{cm}^{-3}$, we paid special attention to very-low-DM candidates, which would normally be dismissed automatically as RFI, carefully checking to see whether they could plausibly be pulsar signals. These low-DM candidates were all at the period of known WSRT RFI birdies, or failed to show up in both the 385 and 1380-MHz data.

\footnotetext{
1 Available at http://www. cv.nrao. edu/ sransom/presto
}

\section{Results and discussion}

No plausible astronomical radio pulsations or bright, dispersed single pulses were detected in any of our observations. Using the radiometer equation modified for pulsar signals (Dewey et al. 1985), we can place limits on 1RXS J141256.0+792204's flux density in these observations (Table 1). We find that 1RXS J141256.0+792204 has a maximum flux density at $400 \mathrm{MHz} S_{400}^{\max } \approx 4 \mathrm{mJy}$ and a maximum flux density at $1400 \mathrm{MHz} S_{1400}^{\max } \approx 0.3 \mathrm{mJy}$, where the fractional uncertainty on these limits is roughly 50\%. RFS07 argue that 1RXS J141256.0+792204 may be a radio pulsar with a nearby distance $d=80-260$ pc. Assuming the pulsar is isolated, we were sensitive to spin periods encompassing the observed range for radio pulsars $\left(P_{\text {spin }} \sim 1 \mathrm{~ms}-10 \mathrm{~s}\right)$, with a factor of roughly 2-5 degredation in sensitivity due to red-noise at the longest periods. Using an assumed distance $d=250$ pc, we can convert our flux density limits to pseudo luminosity $\left(L \equiv S d^{2}\right.$ ) limits. We find $L_{400}^{\max } \approx 0.3(d / 250 \mathrm{pc})^{2} \mathrm{mJy} \mathrm{kpc} \mathrm{kp}^{2}$ and $L_{1400}^{\max } \approx$ $0.03(d / 250 \mathrm{pc})^{2} \mathrm{mJy} \mathrm{kpc}{ }^{2}$. A simple check of the ATNF pulsar catalog $^{2}$ (Manchester et al. 2005) shows that $\lesssim 1 \%$ of the known pulsars have a luminosity below these limits. This suggests that if 1RXS J141256.0+792204 is a radio pulsar, then it is either especially weak, not beamed towards the Earth, or significantly further away than $250 \mathrm{pc}$.

Assuming 1RXS J141256.0+792204 is a radio MSP, what is the probability that its radio beam will pass the Earth (i.e. what is the beaming fraction of such pulsars)? A period-dependent beaming fraction proportional to $P_{\text {spin }}^{-0.5}$ has been shown for normal, un-recycled pulsars (see e.g. Rankin 1993). When extrapolated to millisecond spin periods, this relation implies a $~ 100 \%$ beaming fraction for MSPs. However, Kramer et al. (1998) find observational evidence that this relationship does not apply directly to MSPs and that the beaming fraction of MSPs is more like 50-90\%. These estimates are consistent with considerations of the MSP population in 47 Tuc. Heinke et al. (2005) used a deep Chandra observation of the cluster to perform a population analysis which concluded that there are likely $\sim 25$ radio MSPs ( $<60$ at $95 \%$ confidence) residing in the cluster, independent of beaming. Comparing this with optical and radio studies of the MSP population by Edmonds et al. (2003) and McConnell et al. (2004) respectively, who both conclude that the total MSP population in 47 Tuc is $\sim 30$, Heinke et al. (2005) conclude that the beaming fraction is $\gtrsim 37 \%$. While these various studies suggest that the beaming fraction of MSPs is significantly larger than for normal pulsars, and could be quite high, we cannot strongly rule out beaming as the cause of our non-detection of 1RXS J141256.0+792204.

It is also possible, though we feel unlikely, that we have not seen 1RXS J141256.0+792204 because it is in a compact binary orbit and/or is highly accelerated by a binary companion. Our luminosity limits are for a coherent search and do not include these effects. However, given that we have employed acceleration searches, which are generally sensitive in cases where the total integration time $T_{\text {int }}$ is less than roughly $1 / 10$ of the orbital period $P_{\text {orb }}, 1$ RXS J141256.0+792204 would have to be in a $\$ 2$-h orbit and fairly weak (or eclipsed) not to have been detected in our searches of 15-min data sections. The shortest orbital period of any known radio MSP is $1.6 \mathrm{~h}$ (Camilo et al. 2000, PSR J0024-7204R in the GC 47 Tuc).

\footnotetext{
${ }^{2}$ Available at http://www .atnf.csiro.au/research/pulsar/ psrcat
} 
In conclusion, given the caveats we have discussed, primarily pertaining to extreme orbital or spin parameters, we believe that these searches were sensitive enough to detect any nearby radio pulsar coincident with 1RXS J141256.0+792204, assuming it is beamed towards the Earth. We have checked for catalogued radio sources in the FIRST, NVSS, and WENSS surveys and find no counterpart to 1RXS J141256.0+792204. Future, deeper observations detecting a radio, optical, or X-ray pulsar wind nebula could address whether this source is a nearby radio pulsar beamed away from Earth. Alternate scenarios for 1RXS J141256.0+792204's nature, for instance that it might be the first unhosted CCO (RFS07), should continue to be investigated.

Acknowledgements. J. W. T. H. acknowledges support from an NSERC postdoctoral fellowship and a CSA supplement, tenured at the University of Amsterdam. We would also like to thank Gemma Janssen for help taking the observations. The Westerbork telescope is run by the Dutch radio astronomy institute ASTRON.

\section{References}

Camilo, F., Lorimer, D. R., Freire, P., Lyne, A. G., \& Manchester, R. N. 2000, ApJ, 535, 975

Cordes, J. M., \& Lazio, T. J. W. 2002 [arXiv:astro-ph/0207156]

Dewey, R. J., Taylor, J. H., Weisberg, J. M., \& Stokes, G. H. 1985, ApJ, 294, L25

Edmonds, P. D., Gilliland, R. L., Heinke, C. O., \& Grindlay, J. E. 2003, ApJ, 596, 1197

Heinke, C. O., Grindlay, J. E., Edmonds, P. D., et al. 2005, ApJ, 625, 796

Hessels, J. W. T., Ransom, S. M., Stairs, I. H., Kaspi, V. M., \& Freire, P. C. C. 2007, ApJ, 670, 363

Kramer, M., Xilouris, K. M., Lorimer, D. R., et al. 1998, ApJ, 501, 270

Manchester, R. N., Hobbs, G. B., Teoh, A., \& Hobbs, M. 2005, AJ, 129, 1993

McConnell, D., Deshpande, A. A., Connors, T., \& Ables, J. G. 2004, MNRAS, 348, 1409

Rankin, J. M. 1993, ApJ, 405, 285

Ransom, S. M., Eikenberry, S. S., \& Middleditch, J. 2002, AJ, 124, 1788

Rutledge, R. E., Fox, D. B., \& Shevchuk, A. H. 2007 [arXiv: astro-ph/0705.1011]

Voûte, J. L. L., Kouwenhoven, M. L. A., van Haren, P. C., et al. 2002, A\&A, 385 , 733 